\title{
Impacto social e idoneidad de los servicios de los telecentros españoles en la sociedad de la información: metodología de evaluación a partir de indicadores y método de análisis multivariable
}

\author{
Ana María Morales García \\ Profesora de la Universidad Carlos III de Madrid. Doctora y Master \\ en Documentación. Instituto Universitario Agustín Millares de \\ Documentación y Gestión de la Información de la Universidad Carlos \\ III de Madrid \\ E-mail: amorales@bib.uc3m.es

\section{Mercedes Caridad Sebastián} \\ Profesora de la Universidad Carlos III de Madrid. Doctora en Ciencias \\ de la Información. Instituto Universitario Agustín Millares de \\ Documentación y Gestión de la Información de la Universidad Carlos \\ III de Madrid \\ E-mail: mercedes@bib.uc3m.es
}

Fátima García López

Profesora de la Universidad Carlos III de Madrid. Doctora en Documentación.

Instituto Universitario Agustín Millares de Documentación y Gestión de la Información de la Universidad Carlos III de Madrid

E-mail: fatimag@bib.uc3m.es

\section{Resumen}

Los telecentros españoles constituyen un instrumento de dinamización e inclusión social en la sociedad de la información. Sin embargo, existe una ausencia de indicadores de impacto que permitan evaluar las transformaciones sociales que originan estas entidades en sus áreas de influencia. Teniendo en cuenta este aspecto se formula un sistema de evaluación basado en indicadores y en el método de análisis

multivariable "Rango Selectivo Multidimensional" (RSM) con el objeto de medir el impacto de estas entidades en la sociedad de la información.

\section{Palabras clave}

Telecentros. Evaluación. Indicadores. Métodos de análisis multivariable. RSM.

The social impact and the competence of the spanish telecenters in the Information Society: evaluation methodology from indicators and multivariable methods of analysis

\section{Abstract \\ Spanish telecenters are an instrument for streamlining and social inclusion in the information society. There is, however, a lack of impact indicators that allow for the evaluation of social transformations that originate these entities in their areas of influence. With this in mind, an evaluation sistem with indicators and the "Multidimentional Selective Scale" multivariable methods of analysis as foundations is formulated, with the goal of calculating the impact of these entities in the information society.}

Key-words

Telecenter. Evaluation. Indicators. Multivariable methods of analysis.
1. LOS TELECENTROS ESPAÑOLES: ENTIDADES CLAVE PARA EL DESARROLLO DE LA SOCIEDAD DE LA INFORMACIÓN Y EL CONOCIMIENTO

En la Unión Europea se están desarrollando entidades, cuyo objetivo es eliminar cualquier forma de discriminación social que la introducción de las Tecnologías de la Información y las Comunicaciones puedan generar. Esta idea se materializa a partir del Plan de Acción eEurope e i2010 que establecen, como actuación prioritaria, el desarrollo de un programa de Puntos de Acceso Público a Internet (PAPI), que permita que todos los ciudadanos dispongan en sus municipios de estas entidades, usando preferentemente conexión de banda ancha.

En España, al igual que en el resto de los países, la forma de nombrar a este tipo de entidad varía, tomando diferentes denominaciones que van desde los, ya conocidos, Telecentros, Nuevos Centros del Conocimiento (NCC), Centros de Acceso Público a Internet (CAPI), KZgunea hasta los Puntos de Acceso Público a Internet (PAPI), que amplía su concepto y abarca también a los centros de enseñanza y a las bibliotecas. Las razones están muy ligadas a los servicios que prestan, las actividades desarrolladas y a una forma de denominación local.

- Telecentros: Es el término más difundido y la manera más común de denominar a estas entidades. Proliferan en España a partir de 1991 bajo la ayuda de iniciativas comunitarias, programas y planes de acción europeos financiados por los Fondos Estructurales. Sus metas no se circunscriben a la alfabetización digital, sino que suelen cubrir también servicios de formación ocupacional, asesoramiento, tanto a PYMEs como al público en general, y desarrollo de nuevas formas de trabajo a través de proyectos de teletrabajo. Son fundamentales en zonas rurales o mal comunicadas, pues contribuyen, de forma significativa, a mejorar su entorno más próximo. 
Impacto social e idoneidad de los servicios de los telecentros españoles en la Sociedad de la Información: metodología de evaluación a partir de indicadores y método de análisis multivariable

- Nuevos Centros del Conocimiento (NCC): El proyecto NCC nació, en 1999, con el objetivo de desarrollar el Plan de Alfabetización Tecnológica de Extremadura. Los NCC, generalmente, están ubicados en centros culturales o sociales, donde se ha instalado un equipamiento informático para que todos los sectores de la población adulta tengan acceso a las nuevas oportunidades que las tecnologías están generando en Extremadura.

- Centros de Acceso Público a Internet (CAPI): Las actividades de los CAPI se fomentan a partir del año 2002, financiadas por los Fondos Estructurales y/o iniciativas regionales. Están abiertos a todo el tejido social de los municipios, teniendo en cuenta aquellos grupos que tienen mayor dificultad para el acercamiento a las TIC, a través de actividades formativas (presenciales $\mathrm{u}$ on-line) y talleres de Internet. En menor medida, ofrecen servicios de asesoramiento personalizado y formación como teletrabajadores.

- KZgunea ${ }^{1}$ : Puesto en marcha en 2001, consiste en la creación de una red de centros públicos gratuitos para la formación y el uso de las nuevas TIC, a través de la colaboración del Gobierno Vasco con la Asociación de Municipios Vascos (EUDEL). Los KZgunea ponen Internet al alcance de toda la población de Euskadi, centrando su labor en los sectores más alejados de las nuevas tecnologías: amas de casa, jubilados, desempleados e inmigrantes.

- Puntos de Acceso Público a Internet (PAPI): Es el término más genérico que se aplica también a bibliotecas y centros de enseñanza. Tal y como se ha expresado anteriormente, esta terminología, ha sido acuñada a través de los Planes de Acción eEurope e i2010. A través de los PAPI los ciudadanos deben tener un fácil acceso a Internet en sus municipios, preferentemente a través de banda ancha. Para ello se plantea que los Estados Miembros utilicen los Fondos Estructurales, apoyados con fondos regionales y/o nacionales.

Características generales de los telecentros españoles²:

a) El telecentro es un lugar físico que dispone de medios avanzados en cuanto a las Tecnologías de Información y las Comunicaciones (TIC), cuyo objetivo fundamental

\footnotetext{
${ }^{1}$ KZgunea. [en línea]. [Consulta: 1 de septiembre de 2008]. Disponible en web: <http://www.kzgunea.net/>

2 Morales García, Ana María. Tesis Doctoral. Sociedad de la información en España: el teletrabajo como acción clave [en línea]. Universidad Carlos III de Madrid : Madrid, 2002. Disponible en web: <http://e-archivo.uc3m.es/dspace/handle/10016/481>
}

es la difusión de servicios telemáticos, que se encuentran al alcance de todos y están destinados al desarrollo individual, social y económico del público en general.

b) En la mayoría de los casos, los telecentros españoles son fruto de proyectos o iniciativas, nacionales y europeas, que pueden continuar su labor gracias a la ayuda de instituciones locales o a la colaboración con empresas privadas.

c) La formación es uno de los servicios que encontramos siempre implícito en los telecentros. De hecho, frecuentemente, ellos actúan en sus inicios sólo como centros de aprendizaje, con cursos dirigidos especialmente a fomentar el conocimiento de las Tecnologías de la Información y las Comunicaciones, lo cual supone para la población, generalmente menos favorecida, un primer paso para unirse a la llamada Sociedad de la Información.

d) El telecentro es una solución puente para emprendedores, autónomos y PYMEs que permite adaptar también a la empresa a la nueva Sociedad de la Información, disfrutar de sus instalaciones y de su infraestructura, comenzar a utilizar Internet en los negocios y desarrollar aplicaciones de teletrabajo, fuera o dentro del propio telecentro.

e) El telecentro puede constituir una fuente de empleo, a través de la promoción de teleactividades, poniendo a disposición de los habitantes de la zona módulos dónde pueden desarrollar su actividad. De esa manera, favorece la permanencia de la población autóctona y atrae a los ciudadanos con un alto nivel de formación de las ciudades cercanas.

\section{EVALUACIÓN DEL IMPACTO DE LOS TELECENTROS EN LA SOCIEDAD DE LA INFORMACIÓN}

Los servicios y recursos que ofrecen los telecentros constituyen una parte fundamental de los portales que los identifican en Internet. Sin embargo, en la actualidad, los presupuestos de que disponen estas entidades no han crecido proporcionalmente al incremento de las necesidades de sus usuarios potenciales, por lo que se hace cada vez más necesario la utilización de instrumentos que permitan evaluar los contenidos que se difunden en la Red, con el fin de ayudarles a desarrollar los servicios más necesarios para los usuarios, definirlos y gestionarlos de una manera más efectiva y proporcionar un marco de actuación para los planes y 
políticas de desarrollo de nuevos contenidos regionales, nacionales y, sobre todo, cooperativos.

Partiendo de la base de que la evaluación es la valoración de la utilidad y de la pertinencia de los servicios y recursos prestados por los telecentros a sus usuarios, nos proponemos definir una serie de indicadores que nos permitan, en un futuro, medir su uso y formular el método de análisis multivariante denominado "Rango Selectivo Multidimensional" (RSM), con el objetivo de obtener una evaluación más precisa, pues permite combinar todos los datos aportados por las diferentes variables y obtener un solo valor.

\section{METODOLOGÍA DE TRABAJO}

La primera parte de este estudio "Impacto social e idoneidad de los servicios en los telecentros: estado del arte y modelización de recursos y servicios" ${ }^{3}$ consistió en la búsqueda, identificación y valoración de recursos y servicios destinados a usuarios que visitan los telecentros o, simplemente, para aquellos que buscan información a través de sus portales en Internet.

En una segunda etapa, procedimos a agruparlos en grandes categorías y, finalmente, nos dimos a la tarea de contabilizar los diferentes items por frecuencia de aparición. El resultado obtenido fue la identificación de 85 servicios o recursos, clasificados en 11 categorías, donde destacaron como los más solicitados: la formación y eformación (100\%), todo lo relacionado con Internet (64\%) y los servicios de información al ciudadano (45\%) (gráfico 1).

Una vez tipificados e identificados los servicios prestados por los telecentros, nos planteamos ampliar este estudio con el objetivo de proponer un sistema de evaluación basado en indicadores y en un análisis multivariable denominado Rango Selectivo Multidimensional.

\subsection{Indicadores de evaluación}

A la hora de definir los indicadores se nos planteó la alternativa entre el desarrollo de nuevos indicadores o la adaptación de estándares ya disponibles. Después de un análisis exhaustivo consideramos conveniente aplicar los indicadores ya aceptados internacionalmente

\footnotetext{
3 "Impacto social e idoneidad de los servicios en los telecentros: estado del arte y modelización de recursos y servicios" es un proyecto que se está llevando a cabo en el Instituto Universitario "Agustín Millares", a partir de la "I Conferencia Internacional Brecha Digital e Inclusión Social”, celebrada en Costa Rica, en febrero de 2008.
}

(utilizados, tanto en las bibliotecas, como para medir el impacto en la Sociedad de la Información) y optar por el desarrollo de nuevos indicadores, sólo, en los casos en los que fuera estrictamente necesario.

Para este estudio tomamos como referencia las siguientes normas:

- ISO 2789: 2003. Información y documentación. Estadísticas de bibliotecas para uso internacional (correspondencia Norma Europea EN ISO 2789 de febrero de 2003)

- ISO 5127: 2001 Información y documentación. Vocabularios

- ISO 11620/Amd. 1:2003: Información y documentación. Indicadores para servicios bibliotecarios electrónicos

- UNE 50137: 2000: Información y documentación. Indicadores de rendimiento bibliotecario. (equivale a ISO 11620 de abril de 1998)

- UNE-ISO/TR 20983: 2003 Información y documentación. Indicadores para servicios bibliotecarios electrónicos (julio 2006)

- Indicadores eEurope ${ }^{4}$

- Indicadores $\mathrm{i} 2010^{5}$

- Indicadores Fundación Esplai - Fundación Auna ${ }^{6}$

3.1.1. Indicadores de evaluación: objeto y campo de aplicación

Los indicadores que proponemos ofrecen a los responsables políticos unas directrices para evaluar los recursos y servicios que ofrecen los telecentros en la actualidad. El resultado de esta evaluación permitirá que cada uno de los telecentros elabore su correspondiente plan de mejoras, participe en el desarrollo de un catálogo de buenas prácticas y fomente la colaboración entre las redes y sistemas (figura 1 ).

${ }^{4}$ eEurope benchmarking. [en línea]. [Consulta: 1 de septiembre de 2008]. Disponible en web: <http://ec.europa.eu/ information_society/eeurope/2005/all_about/benchmarking/ index_en.htm>

5 i2010 benchmarking framework. [en línea]. [Consulta: 1 de septiembre de 2008]. Disponible en web: <http://ec.europa.eu/ information_society/eeurope/i2010/docs/benchmarking/ 060220_i2010_benchmarking_framework_nov_2006.doc>

${ }^{6}$ Fundación Esplai-Fundación AUNA. Elaboración de un catálogo de servicios para fomentar el uso de Internet y las nuevas tecnologías. Metodología de trabajo. 2003. [en línea]. [Consulta: 1 de septiembre de 2008]. Disponible en web: 〈http://fomento.telecentros.es/doc/ catalogo.pdf>. 
Impacto social e idoneidad de los servicios de los telecentros españoles en la Sociedad de la Información: metodología de evaluación a partir de indicadores y método de análisis multivariable

GRÁFICO 1

Porcentaje de recursos y servicios ofrecidos por los telecentros

Fuente: Elaboración propia

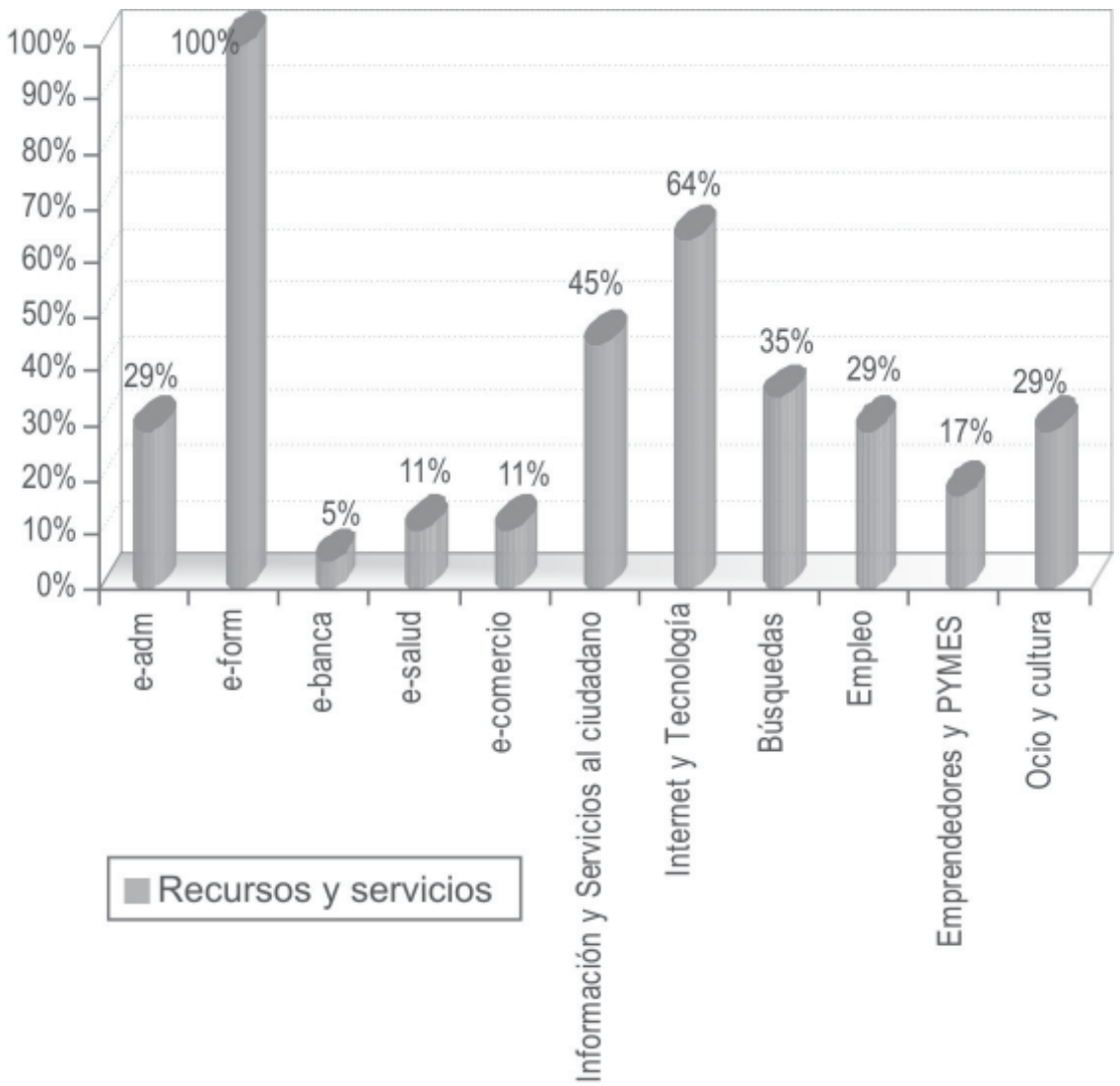

FIGURA 1

Ciclo de evaluación a partir de la propuesta de indicadores y método multivariante Fuente: Elaboración propia

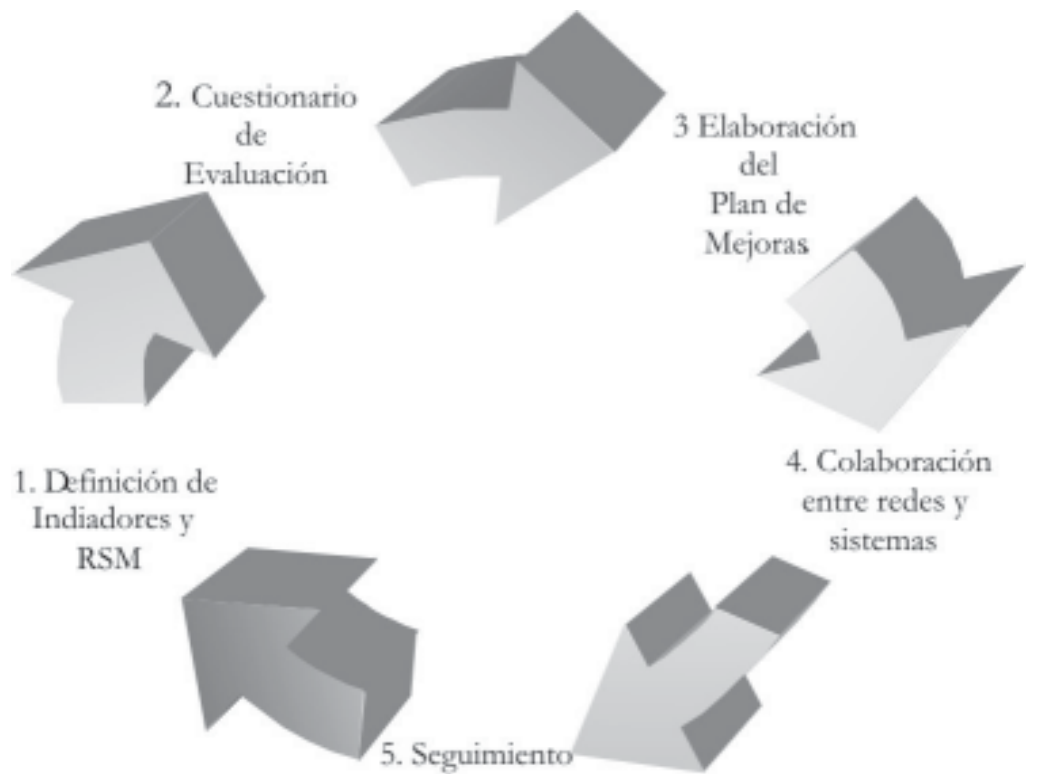

Ci. Inf., Brasília, v. 37, n. 2, p. 70-86, maio/ago. 2008 


\subsubsection{Indicadores de evaluación: términos y definiciones}

Para el establecimeinto de los indicadores de evaluación se aplican los siguientes términos y definiciones?

\begin{tabular}{|c|c|}
\hline Accesil & $\begin{array}{l}\text { Posibilidad de acceso a la información de Internet para todas las personas, independientemente del tipo del } \\
\text { hardware, software, infraestructura de red, idioma, cultura, localización geográfica y capacidades de las } \\
\text { mismas. }\end{array}$ \\
\hline decuación: & rado idoneidad de un indicador para evaluar una actividad específica. \\
\hline isponibilidad: & Telecentro proporciona servicios instalaciones o servicios a los usuarios en el momento qu \\
\hline icacia: & Medida del grado de cumplimiento de los objetivos. \\
\hline ficiencia: & Medida de la utilización de los recursos necesarios para alcanzar un objetivo. \\
\hline raluación: & Estimación de la eficacia, eficiencia, utilidad y relevancia de un servicio o instalación. \\
\hline & Horas a la semana en las que los servicios presenciales del telecentro están disponibles para los usuarios. \\
\hline dicador: & $\begin{array}{l}\text { Expresión (que puede consistir en una serie de números, símbolos o palabras) utilizada para describir } \\
\text { actividades (sucesos, objetos y personas) en términos cuantitativos y cualitativos, para evaluar dichas } \\
\text { actividades, y el método utilizado. }\end{array}$ \\
\hline stalaciones: & Equipos, puestos de trabajo, proporcionados a los usuarios del telecentro. \\
\hline arámetro: & Dato o factor que se toma como necesario para analizar o valorar una situación ${ }^{8}$ \\
\hline $\begin{array}{l}\text { uesto de } \\
\text { abajo: }\end{array}$ & Puestos ofrecidos a los usuarios, con o sin equipo, para trabajar, acceder a Internet, etc. \\
\hline & te caso, solo las fuentes de información electrónicas. \\
\hline ed: & $\begin{array}{l}\text { lectadas entre sí, normalmente a través de un servidor y que puede } \\
\text { ación. }\end{array}$ \\
\hline ervi & efrece de manera presencial o virtual a través de la Red. \\
\hline Telecentro: & $\begin{array}{l}\text { Es el término más difundido y la manera más común de denominar a estas entidades. Sus metas no se } \\
\text { circunscriben a la alfabetización digital, sino que suelen cubrir también servicios de e-administración, e- } \\
\text { formación, e-banca, e-salud, e-comercio, Internet y tecnología, servicios al ciudadano, búsqueda de } \\
\text { información, empleo, asesoramiento a la población, a emprendedores y a PYMEs, difusión de actividades de } \\
\text { ocio y cultura. }\end{array}$ \\
\hline Usab & $\begin{array}{l}\text { Grado de eficacia, eficiencia y satisfacción con la que usuarios específicos pueden lograr sus objetivos, en un } \\
\text { determinados contextos de uso. }\end{array}$ \\
\hline tent & adanos a los cuales están destinados los recursos y servicios del telecentro. \\
\hline isita: & ndencias del telecentr \\
\hline
\end{tabular}

\footnotetext{
${ }^{7}$ Este apartado ha sido elaborado a partir de la Norma ISO 2789:2003, página 7-17, la Norma UNE-ISO /TR 20983: 2006 IN, página 6-8, Norma UNE 50137:2000, página 5-7 y Norma ISO 9241-11: 1998. Ergonomic requirements for office work with visual display terminals (VDT)s - Part 11 Guidance on usability.

${ }^{8}$ Definición tomada de la RAE. [en línea]. [Consulta: 1 de septiembre de 2008]. Disponible en web: <http://www.wordreference.com/es/ en/frames.asp?es=parámetro>
} 
Impacto social e idoneidad de los servicios de los telecentros españoles en la Sociedad de la Información: metodología de evaluación a partir de indicadores y método de análisis multivariable

\subsubsection{Indicadores de evaluación: marco descriptivo}

Los indicadores propuestos se describen de la siguiente manera:

a) En primer lugar, se especifica un parámetro que hace alusión al servicio, la actividad o el aspecto evaluado. Siguiendo la metodología expuesta por Codina ${ }^{9}$ el parámetro responde a la pregunta qué queremos evaluar. Ejemplo: Usuarios, Servicios y recursos, Adecuación de los servicios a usuarios potenciales, Disponibilidad y acceso de recursos Web, Instalaciones y Relación con otros organismos.

b) A continuación, y dentro de cada uno de estos parámetros, se incluye una serie indicadores. Los indicadores responden a la pregunta, de cómo se evalúa cada parámetro ${ }^{10}$.

Para describir cada uno de estos indicadores proponemos el siguiente marco descriptivo, que precisa su significado y su uso ${ }^{11}$ :

\section{Nombre del indicador: cada indicador se identifica con} un nombre descriptivo e inequívoco

a) Objetivo

b) Alcance o campo de aplicación

c) Definición del indicador

\section{d) Método}

e) Interpretación y factores que afectan al indicador

f) Fuentes consultadas
Cada indicador tiene un objetivo explícito formulado en función de los servicios, actividades o aspectos evaluados.

Indica el período de tiempo, la tipología de usuarios y de telecentros a los que es pertinente aplicar el indicador.

Cada indicador se define respecto a los datos que se han de recoger y/o a la relación que se debe establecer entre los mismos.

Se describe de forma concisa los datos que hay que recopilar y los cálculos que se deben realizar.

Se incluye la información necesaria para interpretar los resultados de la utilización del indicador.En el caso de que sea necesario, se proporciona también información sobre factores internos o externos que puedan afectar a los resultados.

Se proporciona las referencias bibliográficas de la fuente del indicador.En el caso de que sea necesario, se incluye también información más detallada sobre la aplicación del indicador, métodos de recogida y análisis de datos, etc.

\footnotetext{
${ }^{9}$ Codina, Luis. Metodología de análisis y evaluación de recursos digitales en línea. Santiago de Compostela : Consorcio Universitario de Galicia, 2003, 30 p (Documento reprografiado).

10 Ibidem

${ }^{11}$ El marco descriptivo expuesto en este artículo se ha realizado a partir de la Norma UNE 50137: 2000, página 9.
} 
3.1.4. Propuesta de indicadores de evaluación ${ }^{12}$

\section{Usuarios}

\subsection{Porcentaje de la población usuaria a la que llegan los servicios de los telecentros}

a) Objetivo

b) Alcance o campo de aplicación

c) Definición del indicador

d) Método

e) Interpretación y factores que afectan al indicador

f) Fuentes consultadas
Determinar la idoneidad del telecentro, respecto a los usuarios potenciales a los que llegan esos servicios

Cada uno de los telecentros, en distintos períodos de tiempo y por grupos específicos de usuarios que viven en la zona (emprendedores, PYMEs y colectivos con dificultad de inserción laboral y social: personas de la tercera edad, discapacitados, desempleados, inmigrantes, jóvenes y mujeres).

Porcentaje de usuarios potenciales que han utilizado cualquiera de los servicios del telecentro en un período de tiempo determinado.

- En el caso de que el telecentro no cuente con estadísticas de usuarios se realizará, en primer lugar, un cuestionario sobre una muestra representativa de la población a la que se le preguntará si ha visitado el telecentro o ha utilizado sus servicios.

- Si se cuenta con estadísticas de usuarios del telecentro, directamente se calculará el porcentaje de la población usuaria a la que llegan estos servicios:

$$
\underline{\mathrm{A}} \times 100 \%
$$

$\mathrm{B}$

Donde:

A: número de personas que han utilizado el telecentro

$B:$ número total de la población a servir

El resultado puede verse afectado por varios factores: composición demográfica de la población, nivel educativo, condiciones socioeconómicas, disponibilidad de terminales para uso y acceso a Internet o nivel de acceso a la Red desde los domicilios de los usuarios.

- UNE/ISO/TR 20903: 2006 IN, página 16, (B.1.1.1)

- Pautas para el servicio de acceso a Internet en las bibliotecas públicas

Cuestionario

\footnotetext{
${ }^{12}$ Los indicadores descritos en este apartado se han definido a partir de las Norma UNE/ISO, las Pautas para el servicio de acceso a Internet en las bibliotecas públicas y el documento publicado por la Fundación Esplai- Fundación Auna "Elaboración de un catálogo de servicios para fomentar el uso de Internet y las nuevas tecnologías. Metodología de trabajo". [en línea]. [Consulta: 1 de septiembre de 2008]. Disponible en web: 〈http://fomento.telecentros.es/doc/ catalogo.pdf>
} 
Impacto social e idoneidad de los servicios de los telecentros españoles en la Sociedad de la Información: metodología de evaluación a partir de indicadores y método de análisis multivariable

\section{Servicios y recursos}

\begin{tabular}{l}
$\begin{array}{l}\text { 2.1. Porcentaje de servicios públicos disponibles } \\
\text { a través del telecentros }\end{array}$ \\
\hline
\end{tabular}

a) Objetivo

Medir la disponibilidad de servicios que se ofrecen a los usuarios.

b) Alcance o campo de aplicación

Cada uno de los telecentros, en distintos períodos de tiempo y por grupos específicos de usuarios que viven en la zona (emprendedores, PYMEs y colectivos con dificultad de inserción laboral y social: personas de la tercera edad, discapacitados, desempleados, inmigrantes, jóvenes y mujeres con déficit cultural y con dificultades de inserción laboral).

c) Definición del indicador

d) Método

e) Interpretación y factores que afectan al indicador

\section{f) Fuentes} consultadas
Porcentaje de servicios que ofrece el telecentro a sus usuarios potenciales, en un período de tiempo determinado. calculará de la siguiente manera:

$$
\underline{\mathrm{A}} \times 100 \%
$$$$
\text { B }
$$

Donde:

A: número de servicios prestados por el telecentro objeto de estudio

$B$ : número total de servicios

Para determinar el número total de servicios, se le sugiere tomar como referencia el "Modelo de los recursos y servicios" (véase ANEXO 1) 13 y el "Catálogo de usos, servicios y contenidos".

- "Impacto social e idoneidad de los servicios en los telecentros: estado del arte y modelización de recursos y servicios".

- "Catálogo de usos, servicios y contenidos" del Programa de Fomento del Uso de Internet y las Nuevas Tecnologías ${ }^{14}$.
El porcentaje de servicios prestados se

\footnotetext{
13 Este "Modelo de recursos y servicios" es una de las aportaciones del estudio "Impacto social e idoneidad de los servicios en los telecentros: estado del arte y modelización de recursos y servicios.

${ }^{14}$ En este recurso se puede acceder a una ficha guía organizada en doce ámbitos de actividad, estructurada a partir de los usos más relevantes y de interés general para la mayoría de personas. Véase: Programa de Fomento del Uso de Internet y las Nuevas Tecnologías. Disponible en web: 〈http://fomento.telecentros.es/index2.htm>
}

\section{c) Definición del indicador}

d) Método

e) Interpretación y factores que afectan al indicador

f) Fuentes consultadas

\section{$\underline{\mathrm{A}} \times 100 \%$ \\ B}

Donde:

A: número de uso de cada servicio prestado por el telecentro

B: número total de servicios que presta el telecentro

El resultado es un número entre 0 y 100 . Una puntuación alta, indica que se están utilizando intensamente los servicios ofrecidos por el telecentro.

- Cuestionarios 
3. Adecuación de los servicios a usuarios potenciales

\subsection{Satisfacción del usuario}

a) Objetivo

b) Alcance o campo de aplicación c) Definición del indicador

d) Método e) Interpretación y factores que afectan al indicador

f) Fuentes consultadas
Evaluar el grado de satisfacción del usuario respecto a las instalaciones, la actitud del personal y los servicios y recursos, que ofrece el telecentro.

Cada uno de los telecentros, en distintos períodos de tiempo y por grupos específicos de usuarios que viven en la zona (emprendedores, PYMEs y colectivos con dificultad de inserción laboral y social: personas de la tercera edad, discapacitados, desempleados, inmigrantes, jóvenes y mujeres con déficit cultural y con dificultades de inserción laboral).

- Puede medir:

- Actitud del personal

- Instalaciones

- Servicios (e-administración, e-formación, e-banca, e-salud, e-comercio, Internet y tecnología, información y servicios al ciudadano, Internet y tecnología, búsqueda de información, empleo, emprendedores y PYMEs, difusión de actividades de ocio y cultura)

Puntuación media aportada por los usuarios de los telecentros, en una escala de tres puntos (de 1 a 3 ), donde (1) es el valor mínimo.

Se diseña un cuestionario con los servicios y recursos a evaluar y/u otros aspectos (actitud del personal e instalaciones). En el cuestionario pueden incluirse preguntas por tipología de usuarios.

Se selecciona una muestra representativa de usuarios y se les solicita que cumplimenten el cuestionario.El valor promedio de la satisfacción del usuario para cada servicio o aspecto se obtiene de la siguiente manera:

\section{$\underline{\mathrm{A}}$ \\ B}

Donde:

A: suma de puntuaciones indicadas por usuarios para cada servicio

B: número de personas que respondieron a las preguntas

Este indicador para cada servicio o aspecto del servicio, dará como resultado un número entre 1 y 3 , con una cifra decimal.

Las opiniones de los usuarios pueden ser subjetivas, depende mucho de las expectativas y circunstancias de los mismos.

- UNE 50137:2000, página.15, (B.1.1.1)

- Cuestionarios 
Impacto social e idoneidad de los servicios de los telecentros españoles en la Sociedad de la Información: metodología de evaluación a partir de indicadores y método de análisis multivariable

\section{Disponibilidad y acceso a los recursos Web}

\subsection{Adecuación del ámbito de navegación y diseño}

a) Objetivo

Evaluar el ámbito de usabilidad incluyendo aspectos relacionados con el diseño de la página como aquellos que favorecen la facilidad de acceso a la información.

b) Alcance o campo de aplicación

c) Definición del indicador

d) Método

e) Interpretación

f) Fuentes consultadas
Cada uno de los telecentros, en distintos períodos de tiempo y por grupos específicos de usuarios que viven en la zona (emprendedores, PYMEs y colectivos con dificultad de inserción laboral y social: personas de la tercera edad, discapacitados, desempleados, inmigrantes, jóvenes y mujeres con déficit cultural y con dificultades de inserción laboral).

- Puede medir:

- Presencia de mapa Web del sitio

- Disponibilidad de herramientas de búsquedas

- Presentación o página de inicio

- Multilingüe

- Presencia de FAQ

- Acceso para discapacitados

- Disponibilidad de videos (multimedia)

Puntuación sobre cada uno de los elementos a evaluar, teniendo en cuenta dos valores $(0$ y 1$)$, donde 1 significa presencia del elemento a evaluar y 0 significa ausencia.

Se diseña un cuestionario donde se especifica la presencia o ausencia de mapa Web del sitio, la disponibilidad de herramientas de búsquedas, la presentación o página de inicio, la posibilidad de multilingüismo, la presencia de FAQ, el acceso para discapacitados y disponibilidad de videos (multimedia).

Este indicador dará como resultado dos valores, donde 1 significa presencia del elemento a evaluar y 0 significa ausencia.

- Fundación ESPLAI-Fundación AUNA. Elaboración de un catálogo de servicios para fomentar el uso de Internet y las nuevas tecnologías. Metodología de trabajo, página 8.

- Cuestionarios
4.2. Adecuación del ámbito de seguridad y privacidad

a) Objetivo

Evaluar la adecuación al ámbito de seguridad y privacidad de la información que el usuario intercambia en la Web.

b) Alcance o

Cada uno de los telecentros, en distintos períodos de tiempo y por grupos específicos de usuarios que viven en la zona (emprendedores, PYMEs y colectivos con dificultad de inserción laboral y social: personas de la tercera edad, discapacitados, desempleados, inmigrantes, jóvenes y mujeres con déficit cultural y con dificultades de inserción laboral).

- Puede medir:

- Privacidad/seguridad (disponibilidad de una declaración de privacidad o seguridad posible).

- Advertencia a ciudadano del uso que se le dará a sus datos.

c) Definición del Puntuación sobre cada uno de los indicador elementos a evaluar, teniendo en cuenta dos valores ( 0 y 1$)$, donde 1 significa presencia del elemento a evaluar y 0 significa ausencia.

d) Método $\quad$ Se diseña un cuestionario donde se especifica si el sitio web del telecentro dispone de una declaración de privacidad o seguridad posible y de una advertencia del uso que se le dará a los datos aportados por los usuarios.

e) Interpretación Este indicador dará como resultado dos valores, donde 1 significa que el sitio web dispone de una declaración de privacidad o seguridad posible y de una advertencia del uso que se le dará a los datos aportados por los usuarios y 0 significa ausencia.

\begin{tabular}{l|l}
$\begin{array}{l}\text { f) Fuentes } \\
\text { consultadas }\end{array}$ & $\begin{array}{l}\text { - Fundación ESPLAI-Fundación } \\
\text { AUNA. Elaboración de un catálogo de } \\
\text { servicios para fomentar el uso de Internet } \\
\text { y las nuevas tecnologías. Metodología de } \\
\text { trabajo, página } 10 . \\
\text { Cuestionarios }\end{array}$ \\
\hline
\end{tabular}


4.3. Porcentaje de elementos o mecanismos que facilitan las visitas continuadas al sitio Web del telecentro

a) Objetivo

b) Alcance o campo de aplicación

c) Definición del indicador

d) Método

e) Interpretación El resultado será un número entre 0 y 100. Una puntuación elevada indica que los usuarios utilizan un número amplio de elementos o mecanismos establecidos por los telecentros para fidelizar el acceso a su

f) Fuentes consultadas sitio Web.

- Fundación ESPLAI-Fundación

Evaluar la adecuación al ámbito de fidelización de la información que el usuario intercambia en la Web.

Cada uno de los telecentros, en distintos períodos de tiempo y por grupos específicos de usuarios que viven en la zona (emprendedores, PYMEs y colectivos con dificultad de inserción laboral y social: personas de la tercera edad, discapacitados, desempleados, inmigrantes, jóvenes y mujeres con déficit cultural y con dificultades de inserción laboral).

- Usuarios que reciben alertas (móviles, email, Newsletter)

- Usuarios con email

- Usuarios que descargan programas (imágenes, melodías, etc.)

- Usuarios que utilizan mensajería instantánea

Porcentaje de elementos o mecanismos que facilitan las visitas continuadas al sitio Web del telecentro, en un período de tiempo determinado.

Se calculará el porcentaje de la población usuaria a la que llegan los servicios de los telecentros: alertas, email, Newsletter, descargas de programas y mensajería instantánea.

$$
\underline{\mathrm{A}} \times 100 \%
$$

B

Donde:

A: suma de elementos establecidos por los telecentros para fidelizar el acceso a su sitio web

B: número total de usuarios potenciales

AUNA. Elaboración de un catálogo de servicios para fomentar el uso de Internet y las nuevas tecnologías. Metodología de trabajo, página 10.

UNE-ISO 20983:2006 IN, páginas.16, 19 у 23, (B.1.1.1, B.1.3.1.5, B1.3.5)

\section{Instalaciones}

\subsection{Población por puestos de trabajo}

\begin{tabular}{l|l} 
a) Objetivo & Evaluar la disponibilidad de estaciones de
\end{tabular} trabajo que ofrece el telecentro, en relación con la población a la que presta sus servicios.

b) Alcance o campo de aplicación

Cada uno de los telecentros, en distintos períodos de tiempo y por grupos específicos de usuarios que viven en la zona (emprendedores, PYMEs y colectivos con dificultad de inserción laboral y social: personas de la tercera edad, discapacitados, desempleados, inmigrantes, jóvenes y mujeres con déficit cultural y con dificultades de inserción laboral).Los telecentros pueden hacer cálculos separados por estaciones conectadas, o no, a la Red.

c) Definición del La relación entre la población a la que el indicador telecentro presta sus servicios y el número de estaciones de trabajo accesibles públicamente.

d) Método

Se establece el número de estaciones de trabajo que están disponibles públicamente para los usuarios de los telecentros.La población por estaciones de trabajo de acceso público es:

\section{$\frac{\mathrm{A}}{\mathrm{B}}$}

Donde:

A: población a la que el telecentro presta sus servicios B: número de estaciones de trabajo de acceso público

e) Interpretación Una puntuación alta indica que existen pocos puestos de trabajo disponibles para los usuarios.

f) Fuentes consultadas
- ISO/TR 20983: 2006, página 29, (B.1.6.2) 
Impacto social e idoneidad de los servicios de los telecentros españoles en la Sociedad de la Información: metodología de evaluación a partir de indicadores y método de análisis multivariable

\subsection{Ratio de uso por puestos de trabajo}

a) Objetivo campo de aplicación

\section{c) Definición} del indicador

d) Método

e) Interpretación

Fuentes consultadas
Evaluar la ratio total de uso de las estaciones de trabajo del telecentro, durante un período de tiempo.

Cada uno de los telecentros, en distintos períodos de tiempo y por grupos específicos de usuarios que viven en la zona (emprendedores, PYMEs y colectivos con dificultad de inserción laboral y social: personas de la tercera edad, discapacitados, desempleados, inmigrantes, jóvenes y mujeres con déficit cultural y con dificultades de inserción laboral).

Porcentaje de estaciones de trabajo en uso en el período de la investigación.

Se calcula el uso de las estaciones de trabajo en intervalos, al azar, durante un período de tiempo.

La ratio de uso de las estaciones de trabajo es:

\section{A $\times 100 \%$}

$$
\text { B }
$$

Donde:

A: número se estaciones de trabajo en uso

B: número total de estaciones de trabajo proporcionadas

El resultado es un número entre 0 y 100. Una puntuación alta indica que se están utilizando intensamente los puestos de trabajo.

ISO/TR 20983: 2006, página 30-31, (B.1.6.3)

\section{Relación con otros organismos}

6.1. Porcentaje de entidades u organismo públicos relacionados con el telecentro

a) Objetivo $\quad$ Determinar el nivel de colaboración del telecentro con entidades y organismos públicos.

b) Alcance o campo de aplicación

Cada uno de los telecentros, en distintos períodos de tiempo y por grupos específicos de usuarios que viven en la zona (emprendedores, PYMEs y colectivos con dificultad de inserción laboral y social: personas de la tercera edad, discapacitados, desempleados, inmigran-tes, jóvenes y mujeres con déficit cultural y con dificultades de inserción laboral).

c) Definición Porcentaje de entidades u organismo públicos del indicador que tienen suscrito algún tipo de convenio o colaboración con el telecentro.

d) Método

Se diseña un cuestionario donde se especifica si el telecentro ha suscrito un tipo de colaboración con otra entidad u organismo y la tipología de entidades con las que se relaciona (Bibliotecas, Telecentros o Redes de Telecentros, Centros culturales, Casa de la mujer, Ayuntamiento, etc.)Se calculará el porcentaje de telecentros que colaboran con otras entidades:

\section{A $\times 100 \%$}

B

Donde:

A: número de entidades que colaboran con el telecentro

B: número total de entidades cercanas al telecentro

e) Interpretación

El resultado es un número entre 0 y 100 . Una puntuación alta indica que existe un alto grado de colaboración entre entidades.

f) Fuentes consultadas
Morales García, Ana María; Caridad Sebastián, Mercedes; García López, Fátima. "Los telecentros en la Sociedad del Conocimiento: situación actual y modelo de evaluación” ${ }^{15}$.

\footnotetext{
${ }^{15}$ Morales García, Ana María; Caridad Sebastián, Mercedes; García López, Fátima. "Los telecentros en la Sociedad del Conocimiento: situación actual y modelo de evaluación”. En: I Conferencia Internacional "Brecha Digital e Inclusión Social", celebrada en San José, Costa Rica del 27 al 29 de febrero de 2008, organizada por PROSIC, Programa Sociedad de la Información y Conocimiento de la Universidad de Costa Rica y el Instituto Universitario "Agustín Millares" de la Universidad Carlos III de Madrid.
} 


\subsection{Descripción del método del Rango Selectivo Multidimensional}

Como se planteó al principio de la investigación, y con el objeto de seleccionar las telecentros más idóneos, se propone aplicar el método matemático denominado Rango Selectivo Multidimensional (RSM), para obtener una evaluación más precisa. Este análisis se basa en el método que aplicó originalmente Ivanovich ${ }^{16}$ para determinar el grado de desarrollo económico de las regiones de la entonces República Socialista Federativa de Yugoslavia. Ya en 1990, como resultado de un trabajo de tesis doctoral presentado por Morales-Morejón ${ }^{17}$, se comenzó a aplicar con éxito en Cuba, como un nuevo método multivariable que permite evaluar de una forma más objetiva la idoneidad de las publicaciones periódicas en varias temáticas. Sin embargo, su total utilización se vio limitada por la complejidad y laboriosidad de sus procedimientos y cálculos matemáticos, hasta que en 1992, Morales-García y García-Díaz ${ }^{18}$, lograron automatizar todos sus cálculos, con la creación del programa EVASOFT, diseñado específicamente para calcular los valores del RSM.

Hasta el año 2000, este software puso en manos de los bibliotecarios y documentalistas, una herramienta fiable, rápida y precisa, que como resultado final ofrece una lista ordenada, en forma ascendente, de las publicaciones seriadas y los valores obtenidos al calcular el RSM. La revista que obtenga un valor del RSM, más próximo a 0 , mostrará un mayor grado de idoneidad respecto a otra, lo que nos permite una mejor gestión de la colección.

\section{Resultados del RSM aplicados a revistas de Economía}

Para ilustrar con un ejemplo, hemos seleccionado un estudio efectuado por Morales García ${ }^{19}$, aplicado a las 153 revistas de Economía. Mediante la aplicación de este tipo de análisis, se puede determinar claramente la

${ }^{16}$ Ivanovich, investigador y matemático, desarrolló el método denominado la I-Distancia de Ivanovich para determinar la distancia entre universos estadísticos multidimensionales.

${ }^{17}$ Morales Morejón, M. Aplicación del Análisis Informétrico para la evaluación de los flujos informacionales en el campo de las plagas y enfermedades de los cítricos (Tesis Doctoral). Academia de Ciencias de Bulgaria, Centro de Información Científica, 1990, 193p.

18 Ana María Morales-García y Alberto Díaz García publicaron la descripción del método del RSM y del software, en el artículo "Evasoft 1.0: Sistema automatizado que permite determinar la idoneidad de las publicaciones seriadas sobre la base del Rango Selectivo Multidimensional (RSM)", en la revista Ciencias de la Información, en 1992, vol. 23 ,n 4 , páginas 273-277.

${ }^{19}$ Morales García, Ana María. Método de análisis univariable versus multivariable. Aplicación práctica en revistas de Economía. En: Forinf@, octubre-diciembre de 2000, No:10, p:8-27. idoneidad de las publicaciones seriadas en una temática dada, pues permite obtener un solo valor, combinando todos los datos aportados por las diferentes variables estudiadas (Uso de la colección, Cantidad de SIR que procesan las revistas, Factor de Impacto, Productividad de las revistas y Opinión de expertos).

Como resultado final obtendremos una lista ordenada, en forma ascendente, de las revistas mejor evaluadas y más idóneas dentro de una colección. Si comparamos los valores aportados por el RSM, con los valores de cada análisis univariable, observamos un desplazamiento en cuanto a posiciones de cada una de las variables (tabla 1, p. 71).

\subsubsection{Requerimientos y metodología para trabajar con el RSM aplicado a los telecentros ${ }^{20}$}

Para obtener los valores del RSM es necesario utilizar el programa EVASOF y seguir los siguientes pasos:

a) Definición de las variables previamente establecidas por el investigador para la evaluación.

- Usuarios.

- Porcentaje de la población usuaria a la que llegan los servicios de los telecentros.

- Servicios y recursos.

- Porcentaje de servicios públicos disponibles a través del telecentro.

- Porcentaje de uso de los servicios públicos disponibles a través del telecentro.

- Adecuación de los servicios a los usuarios potenciales.

- Satisfacción del usuario.

- Disponibilidad y acceso a los recursos Web.

- Adecuación al ámbito de navegación y diseño.

- Adecuación al ámbito de seguridad y privacidad.

- Porcentaje de elementos o mecanismos que facilitan las visitas continuadas al sitio Web del telecentro.

- Instalaciones.

- Población por puestos de trabajo.

- Ratio de uso por puestos de trabajo.

- Relación con otros organismos.

- Porcentaje de entidades u organismos públicos relacionados con el telecentro

${ }^{20}$ Para la realización de todos estos cálculos se utilizará el software EVASOF, diseñado y creado por Ana María Morales-García y Alberto Díaz-García. 
Impacto social e idoneidad de los servicios de los telecentros españoles en la Sociedad de la Información: metodología de evaluación a partir de indicadores y método de análisis multivariable

TABLA 1

Posición que ocupan las revistas de acuerdo a los 5 valores más altos obtenidos en cada una de las variables estudiadas y en el análisis del Rango Selectivo Multidimensional

\begin{tabular}{|c|c|c|c|c|c|c|c|c|}
\hline \multirow{3}{*}{ Título } & \multirow{3}{*}{$\begin{array}{c}\text { Lugar de } \\
\text { publicación }\end{array}$} & \multirow{3}{*}{$\begin{array}{c}\begin{array}{c}\text { Método } \\
\text { multivariable }\end{array} \\
\text { RSM }\end{array}$} & \multicolumn{6}{|c|}{ Métodos univariables } \\
\hline & & & \multirow[b]{2}{*}{$\begin{array}{l}\frac{\pi}{y} \\
\frac{0}{8} \\
0 \\
0 \\
0\end{array}$} & \multirow[b]{2}{*}{$\begin{array}{l}\text { 章 } \\
\text { Zे }\end{array}$} & \multicolumn{2}{|c|}{ Opinión de expertos } & \multirow[b]{2}{*}{ 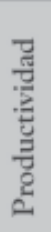 } & \multirow[b]{2}{*}{ 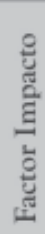 } \\
\hline & & & & & $\begin{array}{l}\frac{7}{0} \\
\frac{\pi}{2} \\
\frac{0}{2} \\
0\end{array}$ & 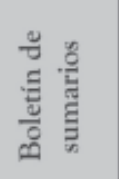 & & \\
\hline $\begin{array}{l}\text { 1. Información } \\
\text { Comercial } \\
\text { Española. Boletín } \\
\text { Económico }\end{array}$ & España & 1 & 1 & & & & 1 & \\
\hline $\begin{array}{l}\text { 2. American } \\
\text { Economic Review }\end{array}$ & USA & 2 & 4 & 2 & 1 & & 6 & 9 \\
\hline 3. The Economist & Reino Unido & 3 & 5 & 7 & & 1 & & 1 \\
\hline 4. Econométrica & Reino Unido & 4 & 3 & & 1 & & & 3 \\
\hline $\begin{array}{l}\text { 5. Journal of } \\
\text { Economic } \\
\text { Literature }\end{array}$ & USA & 5 & 7 & 6 & & & 2 & 2 \\
\hline
\end{tabular}

Fuente: Método de análisis univariable versus multivariable (Morales García, A.M.)

b) Establecimiento del orden de incorporación de las variables

Las variables a considerar tendrán un peso en la evaluación dependiendo del orden que ha decidido el investigador. Esta selección estará sujeta a la importancia que tengan dichas variables. Por ejemplo, se debe introducir como primera la variable aquella cuya información sea la más veraz y objetiva. La variable que en este caso consideramos como la más objetiva sería la "Satisfacción del Usuario".

c) Cálculo del coeficiente de correlación

En este punto es necesario destacar la importancia que tiene una correcta selección de la primera variable que se va a definir como la más importante, pues de ello dependen los resultados totales obtenidos, ya que el mayor peso se le otorga a ella. A partir de ese momento el investigador no controla el orden del resto de las variables. Este orden se determina a partir del cálculo automático del coeficiente de correlación de cada una con respecto a la variable principal, es decir, se coloca en segundo lugar la variable que posea el valor más alto de coeficiente de correlación, y así sucesivamente para cuantas variables se consideren. d) Definir los valores del Telecentro Patrón $\left(\mathrm{P}^{+}\right)$

El “Telecentro Patrón” es el modelo ideal de telecentro, confeccionado con los valores más altos de acuerdo a las variables seleccionadas. Con la elección del método multivariable, denominado Rango Selectivo Multidimensional, los telecentros más idóneos serán los que más se aproximen a los valores establecidos en el Telecentro Patrón, es decir los que se acerquen más a cero en sus resultados. Esta entidad se formará con los valores más favorables de cada una de las variables.

e) Cálculo automático de la desviación típica muestral de cada variable.

f) Cálculo automático del RSM.

Es importante destacar que los valores del Rango Selectivo Multidimensional serán más satisfactorios en la medida que sus resultados se aproximen más cero, lo que significaría una cercanía al "Modelo Ideal de Telecentro". 


\section{CONCLUSIONES}

Los telecentros son instrumentos de dinamización en zonas desfavorecidas. Con esta investigación pretendemos ofrecer una metodología de trabajo para evaluar el uso de los servicios ofrecidos por estas entidades, a través de indicadores y de un método de análisis multivariable, donde se pueden combinar todos los datos por las diferentes variables incluidas en el estudio. Este método pone a disposición de los agentes políticos y sociales un sistema que permite definir la idoneidad de los telecentros y elaborar una adecuada política de desarrollo social.

Artigo submetido em 13/10/2008 e aceito em 22/12/2008.

\section{REFERENCIAS}

CODINA, Luis. Metodología de análisis y evaluación de recursos digitales en linea. Santiago de Compostela: Consorcio Universitario de Galicia, 2003.

EEUROPE una Sociedad de la Información para todos. Disponível em: 〈http://europa.eu/scadplus/leg/es/s21012.htm>. Acesso em: 01 set. 2008.

FUNDACIÓN ESPLAI-FUNDACIÓN AUNA. Elaboración de un catálogo de servicios para fomentar el uso de Internet y las nuevas tecnologias: metodología de trabajo. Disponível em: 〈http://fomento.telecentros.es/doc/ catalogo.pdf>. Acesso em: 01 set. 2008.

I2010: la sociedad de la información y los medios de comunicación al servicio del crecimiento y el empleo. Disponível em: <http:// www.ec.europa.eu/i2010/>. Acesso em: 01 set. 2008.

INDICADORES de acceso para la sociedad de la información: informe mundial sobre el desarrollo de las telecomunicaciones. Ginebra: UIT, 2003. Disponível em: 〈http://www.itu.int/home〉. Acesso em: 01 set. 2008.

INDICADORES para los servicios bibliotecarios electrónicos: (ISO/ TR 20983:2003). Madrid: AENOR, 2006.

MÉTRICA de la sociedad de la información. Madrid: SEDISI, 2000. Disponivel em: 〈http://www.sedisi.es/>. Acesso em: 01 set. 2008.

MORALES GARCÍA, A. M. EVASOFT 1.0.: sistema automatizado que permite determinar la idoneidad de las publicaciones seriadas sobre la base del Rango Selectivo Multidemensional (RSM). Ciencias de la Información, La Habana, v. 23, n. 4, p. 273-277, 1992.

; Ana María; CARIDAD SEBASTIÁN, Mercedes; GARCÍA LÓPEZ, Fátima. Los telecentros en la sociedad del conocimiento: situación actual y modelo de evaluación. In: CONFERENCIA INTERNACIONAL BRECHA DIGITAL E INCLUSIÓN SOCIAL, 1., 2008. San José, Costa Rica. Anales... Costa Rica: Universidad de Costa Rica y Instituto Universitario Agustín Millares de la Universidad Carlos III de Madrid, 2008.

MORALES MOREJÓN, M. Aplicación del análisis informétrico para la evaluación de los flujos informacionales en el campo de las plagas y enfermedades de los cítricos. 1990. Tese (Doutorado)-Academia de Ciencias de Bulgaria, Centro de Información Científica, 1990.

PROGRAMA de Fomento del uso de Internet y las nuevas tecnologías. Disponivel em: 〈http://fomento.telecentros.es/index2.htm〉. Acesso em: 01 set. 2008.

UNE-50137: norma española, información y documentación, indicadores de rendimiento bibliotecario. Madrid: AENOR, 2000.

UNE-EN ISO 2789: información y documentación: estadísticas de bibliotecas para uso internacional. Madrid: AENOR, 2004.

VICENTE, M. R.; LÓPEZ, A. J. Indicadores de la sociedad de la información: una revisión crítica. In: REUNIÓN ASEPELT, 17., 2003, Almería, España. Anales... Almería, España. 2003. Disponível em: <http://www.cibersociedad.net/public/documents/38_bdbr.pdf>. Acesso em: 01 set. 2008. 
Impacto social e idoneidad de los servicios de los telecentros españoles en la Sociedad de la Información: metodología de evaluación a partir de indicadores y método de análisis multivariable

\section{ANEXO 1: MODELO DE RECURSOS Y SERVICIOS}

\begin{tabular}{|c|c|}
\hline Categoría general & Descripción \\
\hline $\begin{array}{l}\text { 1. Administración electrónica } \\
\text { (e-administración) }\end{array}$ & $\begin{array}{l}\text { 1. Gestiones y trámites (hacienda, seguridad social, tarjeta sanitaria, licencias de caza) } \\
\text { 2. Acceso a informaciones sobre leyes y normativas } \\
\text { 3. Atención a inmigrantes, agricultores } \\
\text { 4. Información sobre convocatorias y ayudas } \\
\text { 5. Enlaces a páginas de la e-administración } \\
\text { 6. DNI electrónico }\end{array}$ \\
\hline 2. Formación y e-formación & $\begin{array}{l}\text { 7. Formación para adultos } \\
\text { 8. Formación profesional } \\
\text { 9. Formación de teletrabajadores } \\
\text { 10. Formación para discapacitados } \\
\text { 11. Tutorización para acceso a la universidad de mayores de } 25 \text { años } \\
\text { 12. Cursos para niños } \\
\text { 13. Recursos para docentes, dinamizadores o materiales de apoyo } \\
\text { 14. Orientación escolar } \\
\text { 15. Becas } \\
\text { 16. Idiomas para extranjeros } \\
\text { 17. Concursos, talleres, gymkhanas y juegos en la red }\end{array}$ \\
\hline 3. Banca on-line (e-banca) & $\begin{array}{l}\text { 18. Trámites comuneS } \\
\text { 19. Servicios a PYMEs } \\
\text { 20. Consultas } \\
\text { 21. Información bursátil }\end{array}$ \\
\hline 4. Salud online (e-salud) & $\begin{array}{l}\text { 22. Concertar visitas } \\
\text { 23. Localización de farmacias de guardia } \\
\text { 24. Localización de centros hospitalarios } \\
\text { 25. Telemedicina } \\
\text { 26. Información y servicios sanitarios } \\
\text { 27. Sexualidad } \\
\text { 28. Drogodependencia } \\
\text { 29. Tercera edad } \\
\text { 30. Información sobre asociaciones de apoyo a enfermos } \\
\text { 31. Trámites }\end{array}$ \\
\hline $\begin{array}{l}\text { 5. Comercio electrónico } \\
\text { (e-comercio) }\end{array}$ & $\begin{array}{l}\text { 32. Compra on-line } \\
\text { 33. Información sobre organización de consumidores y usuarios }\end{array}$ \\
\hline $\begin{array}{l}\text { 6. Información y servicios al } \\
\text { ciudadano }\end{array}$ & $\begin{array}{l}\text { 34. Derechos ciudadanos } \\
\text { 35. Información de partidos políticos, sindicatos, ONG } \\
\text { 36. Boletines electrónicos } \\
\text { 37. Estadísticas e informes sobre la Sociedad de la Información } \\
\text { 38. Libros electrónicos } \\
\text { 39. Noticias } \\
\text { 40. Newsletter } \\
\text { 41. Revistas electrónicas } \\
\text { 42. Traducción } \\
\text { 43. Telesecretariado } \\
\text { 44. Gestión de nóminas } \\
\text { 45. Contabilidad } \\
\text { 46. Edición y maquetación } \\
\text { 47. Escaneo } \\
\text { 48. Videoteca } \\
\text { 49. Biblioteca } \\
\text { 50. Sala de reuniones } \\
\text { 51. Asesoría } \\
\text { 52. Glosarios }\end{array}$ \\
\hline
\end{tabular}


ANEXO 1: MODELO DE RECURSOS Y SERVICIOS - CONCLUSIÓN

\begin{tabular}{|c|c|}
\hline Categoría general & Descripción \\
\hline 7. Internet y tecnología & $\begin{array}{l}\text { 53. Chat } \\
\text { 54. Correo electrónico } \\
\text { 55. Creación y desarrollo de weblog } \\
\text { 56. Desarrollo de página Web } \\
\text { 57. Alertas } \\
\text { 58. Foros } \\
\text { 59. Portales turísticos } \\
\text { 60. Software libre (ejemplos: LINEX Y GUADALINEX, aplicación de tiendas virtuales, } \\
\text { búsquedas de empleo, creación de publicaciones digitales, gestión empresarial) } \\
\text { 61. Descargas } \\
\text { 62. Protección a menores }\end{array}$ \\
\hline 8. Búsqueda de información & $\begin{array}{l}\text { 63. Búsquedas en Internet } \\
\text { 64. Localización de información interna } \\
\text { 65. Búsqueda de telecentros } \\
\text { 66. Transporte por carreteras } \\
\text { 67. Medios de comunicación } \\
\text { 68. Consultas lingüísticas } \\
\text { 69. Enciclopedias virtuales }\end{array}$ \\
\hline 9. Empleo & $\begin{array}{l}\text { 70. Información sobre sindicatos } \\
\text { 71. Bases de datos de empresas demandantes } \\
\text { 72. Bolsas de trabajo } \\
\text { 73. Teletrabajo } \\
\text { 74. Derechos laborales } \\
\text { 75. Formación orientada a la inserción laboral }\end{array}$ \\
\hline 10. Emprendedores y PYMEs & $\begin{array}{l}\text { 76. Asesoramiento } \\
\text { 77. Listado de colegios profesionales } \\
\text { 78. Búsquedas de empleo } \\
\text { 79. Bolsa de ideas: gestión y proyectos }\end{array}$ \\
\hline $\begin{array}{l}\text { 11. Difusión de actividades } \\
\text { de ocio y cultura }\end{array}$ & $\begin{array}{l}\text { 80. Agenda de ocio } \\
\text { 81. Museos y exposiciones } \\
\text { 82. Cultura popular } \\
\text { 83. Acceso a portales de ocio } \\
\text { 84. Viajes o información (ejemplo: Camino de Santiago) } \\
\text { 85. Enciclopedia virtual para el rescate del patrimonio cultural (ejemplo: Andalucía } \\
\text { Wikanda) }\end{array}$ \\
\hline
\end{tabular}

